\title{
Release profile and stability evaluation of optimized chitosan/alginate nanoparticles as EGFR antisense vector
}

This article was published in the following Dove Press journal:

International Journal of Nanomedicine

28 June 2010

Number of times this article has been viewed

\author{
Ebrahim Azizi ${ }^{1,4}$ \\ Alireza Namazi' \\ Ismaeil Haririan ${ }^{2,5}$ \\ Shamileh Fouladdel' \\ Mohammad R Khoshayand ${ }^{3}$ \\ Parisa Y Shotorbani ${ }^{6}$ \\ Alireza Nomani ${ }^{1,7}$ \\ Taraneh Gazori ${ }^{1,2}$ \\ 'Molecular Research Lab, Department \\ of Pharmacology and Toxicology, \\ ${ }^{2}$ Department of Pharmaceutics, \\ ${ }^{3}$ Department of Food and Drug \\ Control, Faculty of Pharmacy, Tehran \\ University of Medical Sciences, \\ Tehran, Iran; ${ }^{4}$ Department of Medical \\ Biotechnology, School of Advanced \\ Medical Technologies, Tehran \\ University of Medical Sciences, Tehran, \\ Iran; ${ }^{5}$ Biomaterials Research Center \\ (BRC) Tehran, Iran; ${ }^{6}$ Pharmaceutical \\ Sciences Branch, Azad University, \\ Tehran, Iran; ${ }^{7}$ Department of \\ Pharmaceutics, Faculty of Pharmacy, \\ Zanjan University of Medical Sciences, \\ Zanjan, Iran
}

Correspondence: Taraneh Gazori Department of Pharmaceutics, Faculty of Pharmacy, Tehran University of Medical Sciences, PO Box I4I55-645I,

Tehran, Iran

$\mathrm{Tel}+982 / 44567028$

Fax+982144525410

Email gazori@razi.tums.ac.ir
Abstract: Chitosan/alginate nanoparticles which had been optimized in our previous study using two different N/P ratios were chosen and their ability to release epidermal growth factor receptor (EGFR) antisense was investigated. In addition, the stability of these nanoparticles in aqueous medium and after freeze-drying was investigated. In the case of both N/P ratios $(5,25)$, nanoparticles started releasing EGFR antisense as soon as they were exposed to the medium and the release lasted for approximately 50 hours. Nanoparticle size, shape, zeta potential, and release profile did not show any significant change after the freeze-drying process (followed by reswelling). The nanoparticles were reswellable again after freeze-drying in phosphate buffer with a $\mathrm{pH}$ of 7.4 over a period of six hours. Agarose gel electrophoresis of the nanoparticles with the two different N/P ratios showed that these nanoparticles could protect EGFR antisense molecules for six hours.

Keywords: chitosan/alginate nanoparticles, release profile, freeze-drying, agarose gel electrophoresis

\section{Introduction}

Nanoparticles made from polyelectrolyte complexation have shown potential for use as drug delivery systems. Polyelectrolyte complexes are formed when oppositely charged polyelectrolytes are mixed and interact electrostatically. Chitosan and alginate are polycation and polyanion polyelectrolytes, respectively, that can be used to form a polyelectrolyte complex and to deliver proteins, peptidic drugs, and DNA. ${ }^{1}$

The incorporation of secondary polymers has been one approach used to improve the characteristics of liposome and/or polycation nonviral systems. ${ }^{2}$

Chitosan is a natural linear polysaccharide composed of D-glucosamine and $\mathrm{N}$-acetyl-D-glucosamine units, and is cationic at acidic and neutral $\mathrm{pH}$. Its cationic nature allows it to interact with and easily form complexes with negatively charged DNA.

Chitosan-DNA nanoparticles generally transfect cells more efficiently than naked DNA, but to a lesser extent than commercially available liposome formulations. It has been suggested that the strength of the interaction between chitosan and DNA results in highly stable particles, thereby preventing dissociation within the cell, and ultimately precluding translation of the DNA, resulting in low transfection. Support for this hypothesis comes from studies of the intracellular fate of chitosan-DNA nanoparticles demonstrating that these particles remain intact intracellularly for several days. Formulations facilitating the release of DNA, such as those including hydrophobic moieties or with the addition of an anionic polymer, could improve chitosan-based vector transfection. ${ }^{3}$ 
Alginate is an anionic copolymer of 1, 4-linked $\beta$-D mannuronic acid and $\alpha$-L-guluronic acid residues. In the presence of divalent cations such as calcium, alginate forms a gel, due to the stacking of guluronic acid blocks with the formation of "egg-box" calcium-linked junctions. ${ }^{4}$

In the present study, due to a number of desirable characteristics and the demonstrated success of the alginate-chitosan (Alg/Chi) system, it was decided to explore its development and use in the submicron scale and to evaluate its potential use as a stable carrier during preparation and storage.

For this purpose, $\mathrm{Alg} / \mathrm{Chi}$ nanoparticles were produced based on the formation of a polyionic complex between two biopolymers. Pregel preparation was carried out using alginate and calcium chloride. Chitosan and epidermal growth factor receptor (EGFR) antisense oligonucleotide (EGFR AS) solutions were subsequently added to the pregel for preparing nanoparticles of EGFR AS.

EGFR AS was used to reduce the expression of EGFR, a receptor tyrosine kinase proto-oncogene that plays a central role in the initiation and development of several human malignancies, notably of the breast, brain, and lung. ${ }^{5}$ Based on the results of size and zeta potential optimization of nanoparticles in our previous study, ${ }^{6}$ two different N/P ratios were chosen as the best formulations, and the release profiles of EGFR AS from these two nanoparticles were evaluated.

The macromolecular drug can degrade during preparation of the nanoparticles, during storage, and also during in vitro or in vivo release. For example, the drug may be exposed to heat, shearing forces, $\mathrm{pH}$ extremes, organic solvents, interfaces, freezing, and drying during preparation of the nanoparticles. ${ }^{7}$ Therefore, evaluation of nanoparticle stability is necessary to confirm the suitability of $\mathrm{Alg} / \mathrm{Chi}$ nanoparticles for antisense delivery.

Nanoparticle stability is necessary for protection of extracellular oligonucleotide molecules. Agarose gel electrophoresis has been used to observe the electrophoretic migration behavior and has confirmed that nanoparticles can protect antisense oligonucleotide molecules from dissociation during their preparation and also their exposure to cells. ${ }^{8}$

The stability of antisense-loaded nanoparticles was investigated in aqueous medium (using agarose gel electrophoresis) and after freeze-drying to evaluate the efficacy of nanoparticles as an antisense vehicle. Size, zeta potential, scanning electron microscopy (SEM) images, and release profiles of nanoparticles were compared before and after freeze-drying to determine if there were any changes in these characteristics after freeze-drying.
Chitosan and alginate have high $\mathrm{pH}$-dependent swelling properties, which could be used to control the release of drugs. ${ }^{9,10}$ It is well established that water exists in polymer networks of hydrogels in three different physical states, ie, free water that can freeze at the usual freezing point, intermediate water that freezes at a temperature lower than the usual freezing point, and bond water which cannot freeze at the usual freezing point. Biomedical or pharmaceutical activity depends on how the water molecules associate with the polymer. Moreover, the swelling characteristics of hydrogels are dominated by the nature of the polymer and the state of water. ${ }^{9}$ The ability of the nanoparticles to swell was investigated by evaluation of their size increase in medium buffer after freeze-drying, to ensure that the nanoparticles could be reswelled again in aqueous solution and release their drug content during swelling.

The unchanged characteristics of antisense-loaded Alg/ Chi nanoparticles after freeze-drying and also the ability of these nanoparticles to protect the antisense molecules from degradation in aqueous medium were important reasons to investigate the usefulness of this antisense delivery system.

\section{Materials and methods Materials}

Guluronate-rich sodium alginate (BDH Co., UK) in which the $\mathrm{M} / \mathrm{G}$ ratio and viscosity of a $1.0 \% \mathrm{~W} / \mathrm{V}$ solution is 0.31 and $38 \mathrm{cp}$, respectively, low molecular weight chitosan (SigmaAldrich Co., Germany), and calcium chloride (Merck KGaA Co., Germany), EGFR phosphorothioated 21 mer antisense 5' (TTTCTTTTCCTCCAGAGCCCG) 3' (TIB MOLBIOL ${ }^{\circledR}$ GmbH., Germany) were used.

An optimized formulation of particles was prepared as previously described by Gazori et al by adding chitosanantisense solution to calcium alginate pregel at pre-defined concentrations. ${ }^{6}$

\section{Particle size and zeta potential}

Nanoparticle size and zeta potential were assessed by photon correlation spectroscopy using a Malvern zetasizer/scattering particle size analyzer (Malvern ZS Series Co., UK). To do this, a sample of $2 \mathrm{~mL}$ was vortexed, placed into an analyzer chamber, and measured.

Nanoparticle morphology, such as shape and presence of aggregates, was studied by SEM. Samples of nanoparticles were mounted on metal stubs, coated with platinum under vacuum, and then examined on a LEO1455 VP electron microscope (10 kV, Cambridge). 


\section{Drug release}

The drug release study was performed using the dialysis sack method with $23 \times 15 \mathrm{~mm}$ DO-405 dialysis tubing (Sigma, Germany). Antisense-loaded nanoparticles containing $63 \mu \mathrm{g}$ of antisense were added to the dialysis bag with a molecular cutoff of $10-12 \mathrm{kDa}$. The bag was then sealed and placed in a beaker containing $100 \mathrm{~mL}$ of phosphate buffer (pH 7.4) with an agitation rate of $300 \mathrm{rpm}$ on a magnetic stirrer. Samples were taken at intervals for four days and replaced with fresh phosphate buffer. The samples were analyzed spectrophotometrically at $260 \mathrm{~nm}$, and the release percentage in each interval was calculated. The stability of the nanoparticles after freeze-drying and also in aqueous medium was examined.

\section{Nanoparticle freeze-drying}

Nanoparticle suspensions were frozen at $-20^{\circ} \mathrm{C}$ for 24 hours and then lyophilized in (Christ, Alpha 2-4 Lyophilizator, Germany). The lyophilized particles were resuspended and evaluated for size, zeta potential, shape, and release profile to determine if nanoparticle characteristics were affected by the lyophilization process.

\section{Gel electrophoresis}

A 2\% agarose solution in Tris, acetic acid, and ethylenediaminetetraacetate buffer was prepared, and $0.1 \%$ ethidium bromide was added to the solution to prepare the agarose gel. Nanoparticles with two different N/P ratios $(5,25)$ were produced in three different series. In the first series, nanoparticles were incubated in aqueous medium for six hours; in the second series, nanoparticles were prepared by incubation for three hours; and the third series comprised freshly prepared nanoparticles. The three series of loaded nanoparticles, as well as naked EGFR AS and nanoparticles without EGFR AS, were mixed with loading dye and put into agarose gel wells separately; $6 \mu \mathrm{g}$ of antisense was used in all cases. Samples in agarose gel were then electrophoresed for 60 minutes at $75 \mathrm{~V}$. The resulting gel was viewed under ultraviolet (UV) illumination and photographs were taken using a UV documentation device (Bio Doc-IT System, Upland, CA).

Particle preparation yields were calculated by dividing the weight ratios of the nanoparticles on applied polymers and multiplying by 100 .

After the freeze-drying process, the nanoparticles were placed in phosphate buffered saline ( $\mathrm{pH}$ 7.4) while stirring, and at a series of intervals their size was assessed by a Malvern zeta sizer over eight hours.

\section{Statistical analysis}

For all the results, triplicate readings were obtained for a minimum of three separate samples. For comparison of means before and after the freeze-drying process, paired t-tests were applied to the means \pm standard deviation (SD). A $P$ value $<0.05$ was considered to be significantly different in all cases.

\section{Results and discussion Nanoparticle size}

The optimized formulation which had the smallest size (194 nm), good monodispersity, and good loading efficacy (95.6\%) was chosen on the basis of the results of our previous study. The composition of this formulation was a Chi/Alg ratio of 1 , calcium chloride $0.2 \%$, and $\mathrm{N} / \mathrm{P}$ ratio of 5 . A second formulation had a Chi/Alg ratio of 1 , calcium chloride $0.2 \%$, an N/P ratio of 25, with a particle size of $430 \mathrm{~nm}$ and loading efficacy of $66.5 \%$. Antisense was added to the chitosan solution and this complex was added to the calcium alginate pregel. Oligonucleotide molecules with negative electrostatic force bind to chitosan which has a positive electrostatic force, causing loading of antisense molecules to the $\mathrm{Alg} / \mathrm{Chi}$ nanoparticles. ${ }^{11}$

The second formulation with an N/P ratio of 25 was also compared with the formulation having an N/P ratio of 5 , because in future studies we intend to check the prepared nanoparticles in cell culture with at least two different $\mathrm{N} / \mathrm{P}$ ratios. Therefore, the release capability and stability of these formulations were checked as part of this study (see Table 1).

Table I Nanoparticle size and zeta potential before and after the freeze-drying process

\begin{tabular}{llllll}
\hline & & $\begin{array}{l}\text { Nanoparticles } \\
\text { size }(\mathbf{n m})\end{array}$ & $\begin{array}{l}\text { Zeta potential } \\
(\mathbf{m V})\end{array}$ & $\begin{array}{l}\text { Burst release \% } \\
\text { (up to I5 min) }\end{array}$ & $\begin{array}{l}\text { T I00\% } \\
\text { (h) }\end{array}$ \\
\hline Alg/Chi-AS & BFD $^{\mathrm{a}}$ & $196 \pm 12$ & $30.0 \pm 4.1$ & $58.3 \pm 2.3$ & $50.0 \pm 0.5$ \\
N/P 5 & AFD $^{b}$ & $185 \pm 8$ & $25.0 \pm 5.3$ & $56.5 \pm 2.7$ & $51.0 \pm 1.0$ \\
Alg/Chi-AS & BFD & $430 \pm 21$ & $43.0 \pm 3.3$ & $50.6 \pm 1.6$ & $45.0 \pm 0.7$ \\
N/P 25 & AFD & $425 \pm 26$ & $46.0 \pm 2.7$ & $51.3 \pm 2.1$ & $43.0 \pm 1.2$ \\
\hline
\end{tabular}

Notes: aBefore freeze-drying. ${ }^{b}$ After freeze-drying. 
The preparation yields of nanoparticles having $\mathrm{N} / \mathrm{P}$ ratios of 5 and 25 were $73.4 \% \pm 4.2 \%$ and $62.8 \% \pm 5.4 \%$, respectively. This could be referred to a larger $\mathrm{N} / \mathrm{P}$ ratio of 25 which may cause faster aggregation than an N/P ratio of 5 , resulting in fewer yields.

SEM images showed that nanoparticles have a spherical shape and are monodispersed. The SEM images of freeze-dried nanoparticles which were resuspended and then prepared for SEM imaging also showed particles of the same shape and size with very few aggregates. It seems that the freeze-drying process does not affect the physical characteristics of these nanoparticles. Therefore, this is a good way to store the nanoparticles and increase their shelf-life when they are to be used as a drug-delivery system (Figures $1 \mathrm{~A}$ and $1 \mathrm{~B}$, and Table 1).

\section{Nanoparticle zeta potentials}

Zeta potentials for the two formulations with N/P ratios of 5 and 25 were $30 \pm 4.1 \mathrm{mV}$ and $43 \pm 3.3 \mathrm{mV}$, respectively. It was also shown that there was not any significant difference between the zeta potentials of the two formulations before and after freeze-drying (see Table 1), which indicates the suitability of the freeze-drying process for storage of these nanoparticles.

\section{Ability of nanoparticles to swell}

Freeze-dried nanoparticles with an N/P ratio of 5 were placed in phosphate buffer with a $\mathrm{pH}$ of 7.4, and size analysis showed that the nanoparticles can be reswelled in medium after freeze-drying within approximately six hours and that thereafter the nanoparticles start to degrade, causing a decrease in nanoparticle size (Figure 4). Reswelling is necessary to release the antisense molecules from the hydrogel matrices. ${ }^{9}$

\section{Drug release}

Release profiles of nanoparticles made with two different $\mathrm{N} / \mathrm{P}$ ratios of 25 and 5 showed that nanoparticles can release antisense over 45 and 50 hours respectively, and that there is no significant difference between the release profiles of both nanoparticles before and after freeze-drying. Drug diffusion out of a hydrogel matrix is dependent on the mesh sizes within the matrix of the gel. Typical mesh sizes reported for biomedical hydrogels range from 5 to $100 \mathrm{~nm}$ (in their swollen state), which are much larger than most small-molecule drugs. As a result, diffusion of these drugs is not considerably retarded in the swollen state, whereas macromolecules like oligonucleotides, due to their hydrodynamic radii, will have a sustained release unless the structure and mesh size of the swollen hydrogel are designed appropriately to obtain desired rates of macromolecular diffusion. In the case of the swelling-controlled mechanism, when diffusion of a drug is significantly faster than hydrogel distension, swelling is considered to be controlling the release behavior. Finally, chemically controlled release is determined by chemical reactions occurring within the gel matrix. These reactions
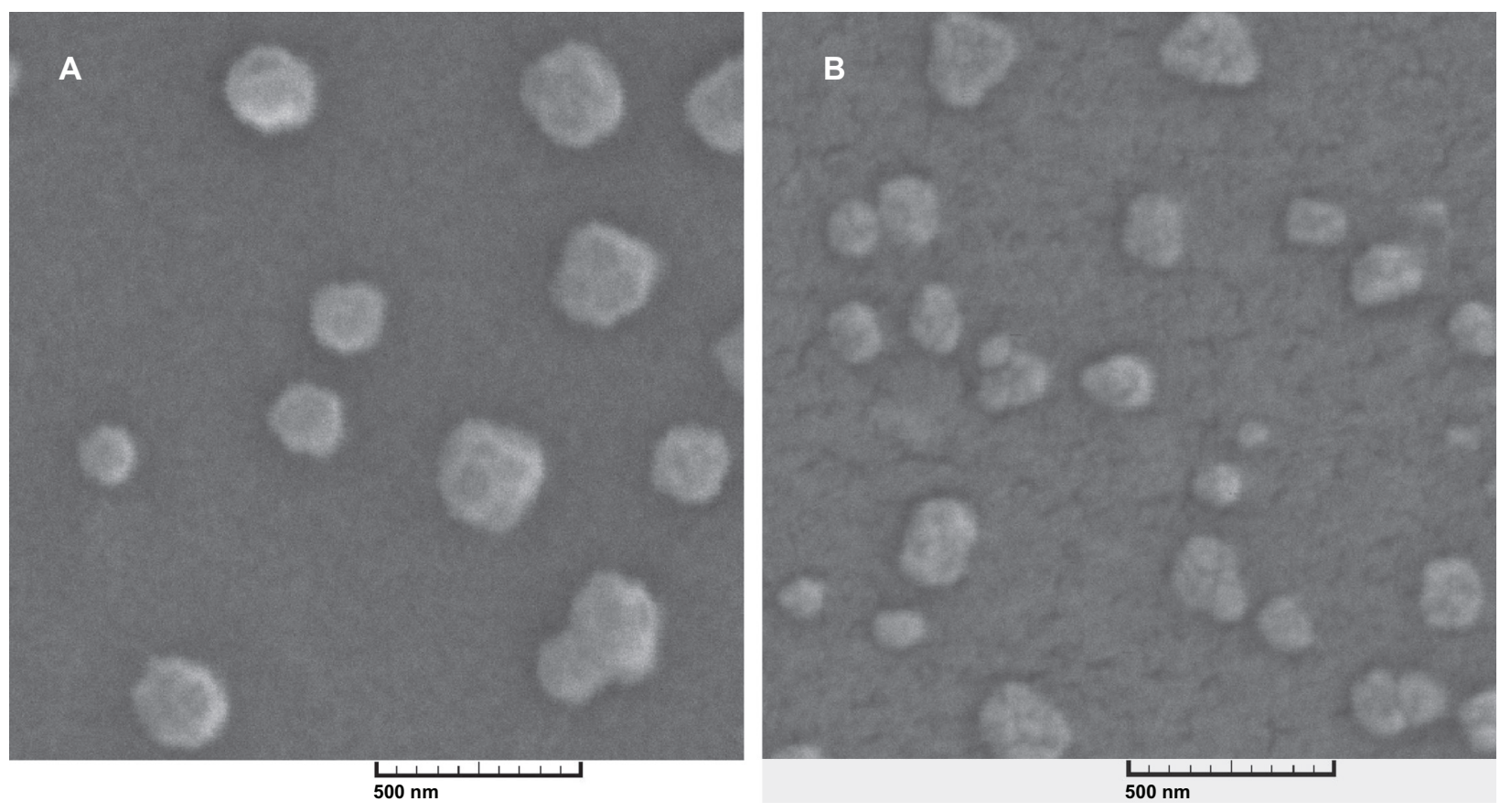

Figure I Scanning electron microscopy image of alginate-chitosan nanoparticles with an N/P ratio of 5 before (A) and after (B) the freeze-drying process, magnification $50 \times$. 
include polymeric chain cleavage via hydrolytic or enzymatic degradation, or reversible/irreversible reactions occurring between the previously described release mechanisms. Under certain circumstances, surface or bulk erosion of hydrogels or the binding equilibrium among the drug-binding moieties incorporated within the hydrogels are two different mechanisms reported as controlling the rate of drug release (Figure 2). ${ }^{12}$

Douglas et al reported that $\mathrm{Chi} / \mathrm{Alg}$ nanoparticles loaded by DNA with two different N/P ratios $(10,25)$ released $6 \%$ and $3.5 \%$ of adsorbed DNA, respectively, in PBS (phosphatebuffered saline) over 48 hours. $^{2}$

Borges et al reported that alginate can increase the stability of chitosan nanoparticles and reduce the release rate of ovalbumin from $\mathrm{Alg} / \mathrm{Chi}$ nanoparticles.${ }^{13}$ In another study, ${ }^{14}$ colloidal complexes of antisense oligonucleotide with poly-L-lysine (PLL) and protamine salts were formed. Release profiles of antisense oligonucleotide were studied in water and $\mathrm{PBS}$ at $\mathrm{pH}$ 7.4. It was reported that the dissociation behavior of antisense with PLL or protamine was better enhanced in PBS than in deionized water. The phosphate anions probably compete with the oligonucleotide to occupy the positive polycation groups and this causes dissociation of antisense molecules from polycations. $^{14}$

The release profiles of the formulations investigated in the present study were not affected by freeze-drying (Figures $2 \mathrm{~A}$ and $2 \mathrm{~B}$ ), which is essential for a good drug delivery system,

A

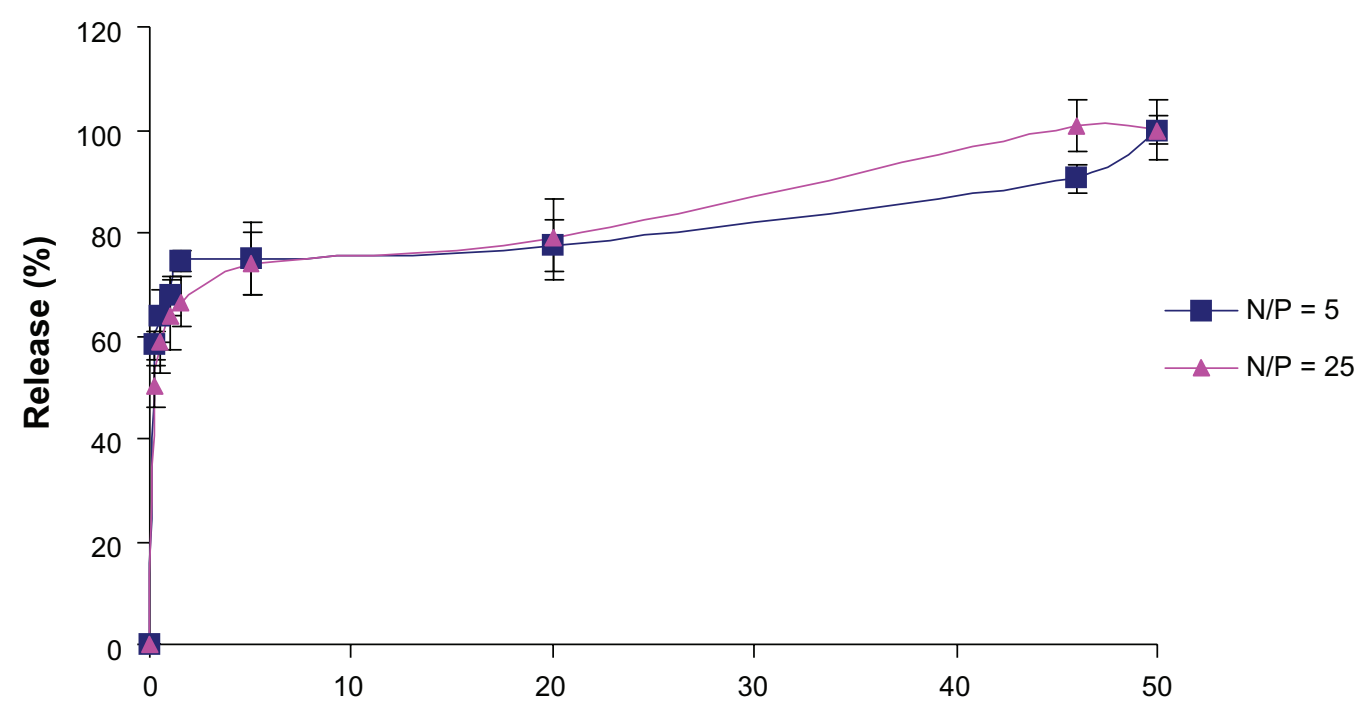

B

Time (h)

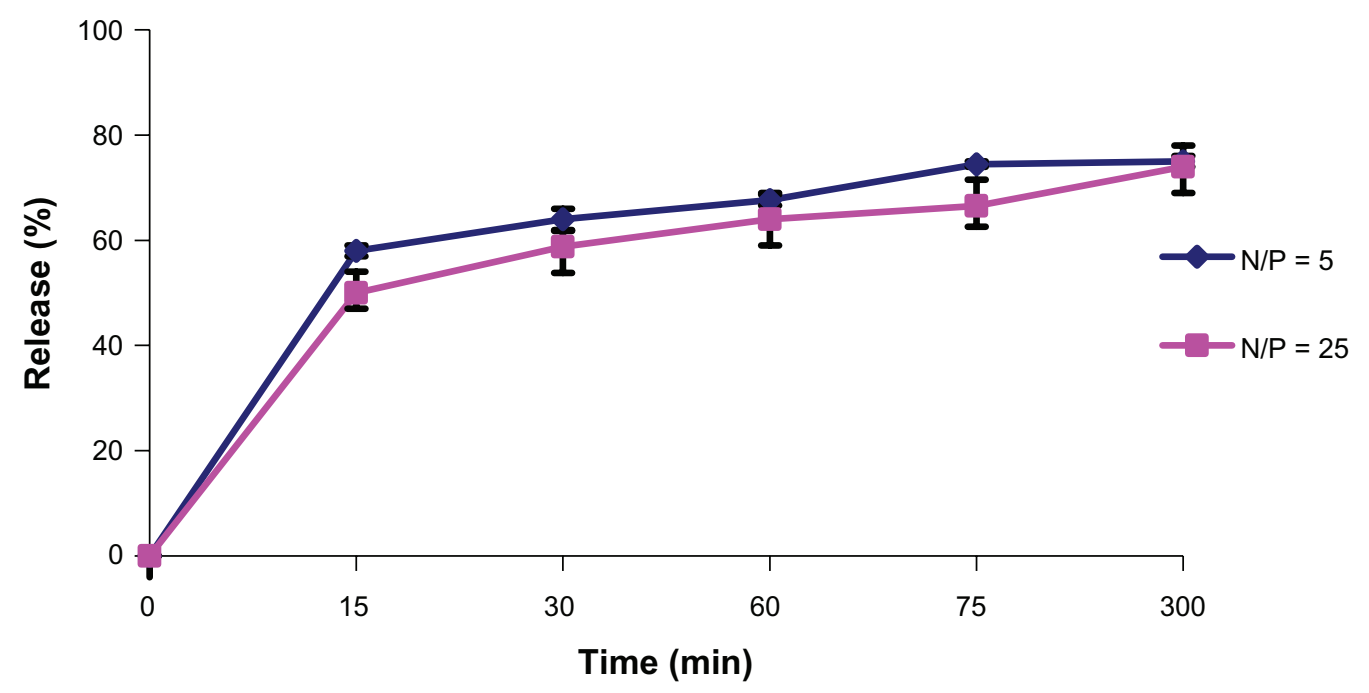

Figure 2 Release profile of nanoparticles with N/P ratio of 5 and 25 (A) over 50 hours and (B) in the first six hours. 


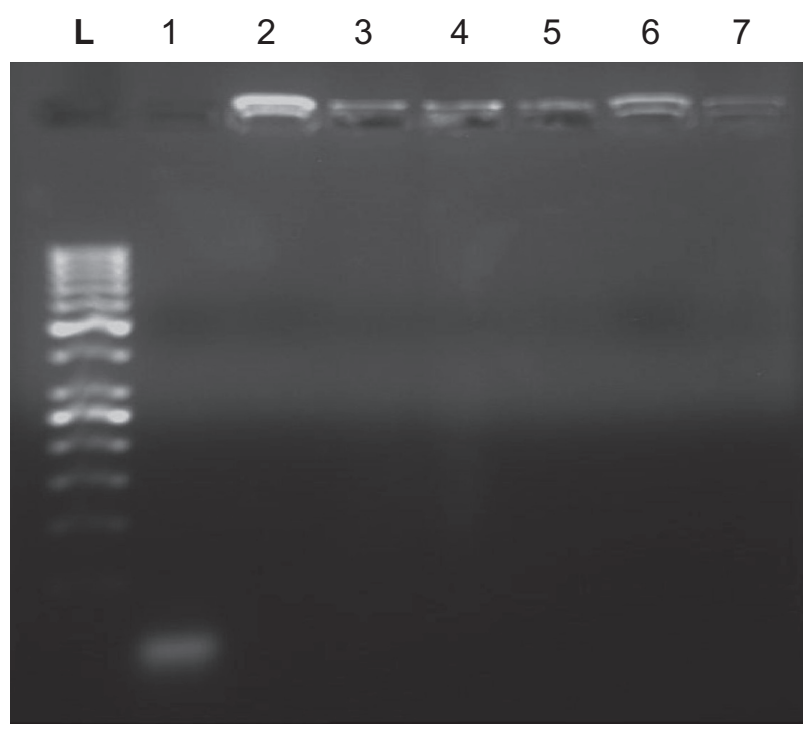

Figure 3 Gel electrophoresis analysis of alginate-chitosan nanoparticles. L (ladder), I (naked AS), 2 (N/P 5, one hour), 3 (N/P 5, three hours), 4 (N/P 5, three hours), 5 (N/P 25, one hour), 6 (N/P 25, three hours), 7 (N/P 25, six hours). The image has been taken after staining by ethidium bromide and visualized under ultraviolet light.

thereby protecting the drug until it is released at its target region (see Table 1).

There is a significant difference between the percentage of burst release from nanoparticles with $\mathrm{N} / \mathrm{P}$ ratios of 5 and 25 for up to 15 minutes in PBS (Table $1, P \leq 0.001$ ), ie, the burst release from nanoparticles with an $\mathrm{N} / \mathrm{P}$ ratio of 25 is significantly lower than that from those with an N/P ratio of 5 . This difference might be due to nanoparticle size. Nanoparticles with an N/P ratio of 5 are smaller than those with a ratio of 25 , so antisense molecules have not enough space to diffuse through these smaller nanoparticles and therefore remain on the surface. Also, there is a greater ionic interaction force between nanoparticles with an N/P ratio of 25 than nanoparticles with a ratio of 5 , so release could be faster in nanoparticles with an N/P ratio of 5 in PBS buffer. On the other hand, T 100\%, which is the time necessary to reach the release percentage at steadystate, is $50 \pm 0.5$ and $45 \pm 0.7$ hours in nanoparticles with $\mathrm{N} / \mathrm{P}$ ratios of 5 and 25 , respectively. There is a significant difference in release between these time points $(P \leq 0.001)$, which means that nanoparticles with an N/P ratio of 5 can release antisense molecules at a slower rate than those with an N/P ratio of 25 as time passes, which could be due to faster erosion of the larger nanoparticles because of their greater surface area.

\section{Drug stability}

The stability of many colloidal systems is directly related to the magnitude of their zeta potential. In general, if the value of the particle zeta potential is large, the colloidal system will be stable. Conversely, if the particle zeta potential is relatively small, the colloidal system will become agglomerated. The surface charge of the particles is of substantial importance in all the production steps of these coated particles because the efficiency of the different steps is directly related to electrostatic interactions. ${ }^{13}$

$\mathrm{Alg} / \mathrm{Chi}$ nanoparticles showed a positive zeta potential value of about $+30 \mathrm{mV}$ (see Table 1), which indicates good stability of the particle suspensions in aqueous medium. Nanoparticle size, zeta potential, and dissolution profiles were compared between freshly produced particles and those which had been resuspended following freeze-drying. SEM images showed that freeze-dried nanoparticles could swell again in aqueous medium and there was no significant difference before and after the freeze-drying process.

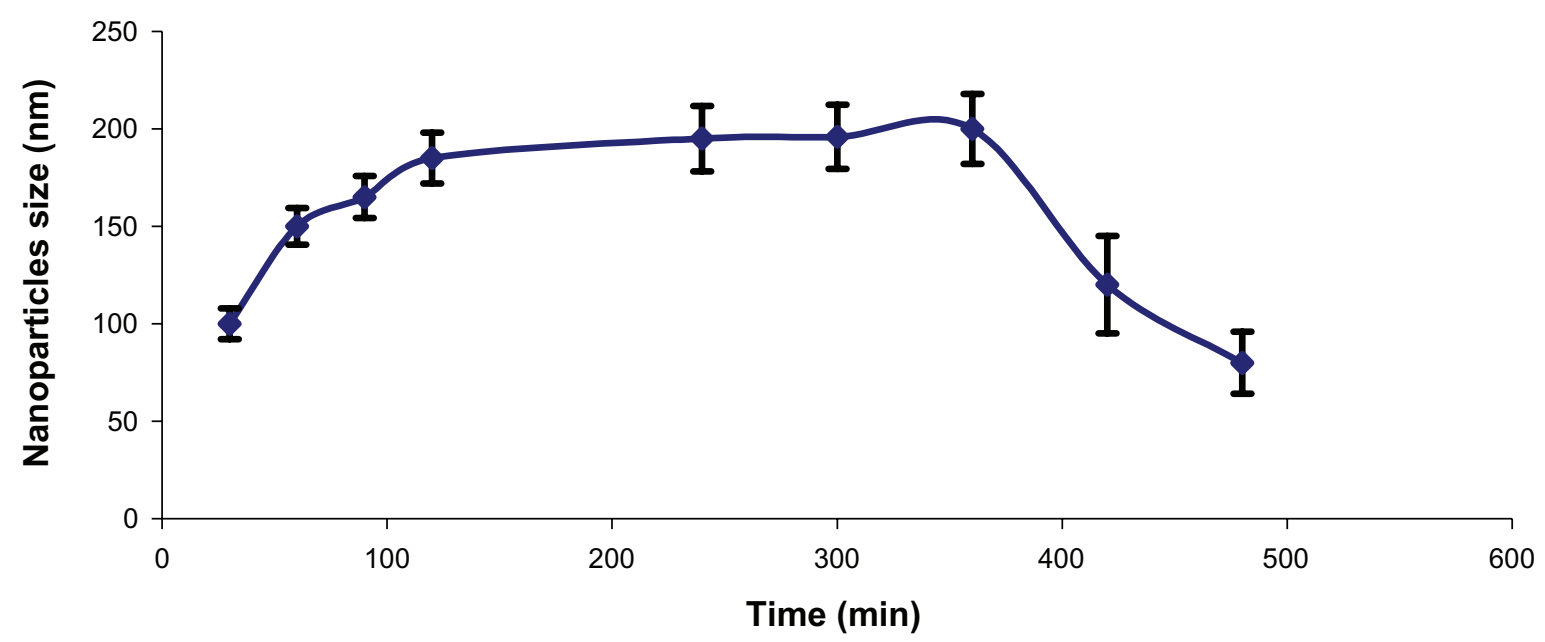

Figure 4 Swellability of antisense-loaded alginate-chitosan nanoparticles with an N/P ratio of 5, in phosphate-buffered saline at $\mathrm{pH} 7.4$. 
So lyophilization can be used as a suitable method for the storage of these nanoparticles. ${ }^{15,16}$

Gel electrophoretic evaluation of nanoparticles incubated in aqueous medium showed that both nanoparticles with N/P ratios of 5 and 25 retained the antisense molecules, even after six hours of incubation, and remained intact in the wells. This indicates that the nanoparticles can retain and stabilize the content of antisense in their hydrogel matrix, although naked antisense migrated towards the bottom of the gel as a result of its low molecular weight (6300 Da) (Figure 3).,17

\section{Conclusions}

Stability evaluation of freeze-dried Alg/Chi nanoparticles showed that this process does not change the characteristics of the nanoparticles and suggests that they could be a good storage method. Furthermore, nanoparticles can protect antisense molecules during the preparation and treatment periods. The release profile of EGFR antisense showed that these molecules can be released from nanoparticles over 50 hours, and approximately $70 \%$ of the release occurred in the first five hours. These findings indicate that $\mathrm{Alg} / \mathrm{Chi}$ nanoparticles may be good carriers for the delivery of antisense oligonucleotides.

\section{Acknowledgments}

The authors appreciate the financial support of the Office of the Vice-Chancellor for this research (Grant No. 4206). Thanks are extended to Dr Sajjadi and Dr Yoosefi for their kind help with the preparation of this manuscript.

\section{Disclosure}

The authors report no conflict of interest in this work.

\section{References}

1. Sarmento B, Ferreira D, Veiga F, Ribeiro A. Characterization of insulin-loaded alginate nanoparticles produced by ionotropic pre-gelation through DSC and FTIR studies. Carbohydrate Polymers. 2006;66:1-7.

2. Douglas KL, Tabrizian M. Effect of experimental parameters on the formation of Alginate-Chitosan nanoparticles and evaluation of their potential application as DNA carrier. J Biomater Sci Polym Ed. $2005 ; 1: 43-56$.
3. Douglas KL, Piccirillo CA, Tabrizian M. Effects of alginate inclusion on the vector properties of chitosan-based nanoparticles. J Control Release. 2006;115:354-361.

4. Hollins AJ, Benboubetra M, Omidi Y, et al. Evaluation of generation 2 and 3 poly (propylenimine) dendrimers for the potential cellular delivery of antisense oligonucleotides targeting the epidermal growth factor receptor. Pharm Res. 2004;21:458-466.

5. Silva CM, António J, Ribeiro A, Figueiredo M, Ferreira D, Veiga F. Microencapsulation of hemoglobin in chitosan-coated alginate microspheres prepared by emulsification/internal gelation. AAPS J. 2006;7:E903-E913.

6. Gazori T, Khoshayand MR, Azizi E, Yazdizade P, Nomani A, Haririan I. Evaluation of alginate/chitosan nanoparticles as antisense delivery vector: Formulation, optimization and in vitro characterization. CarbohydratePolymers. 2009;77:599-606.

7. Riaz AA, Bodmeier R. Preparation of performed porous PLGA microparticles and antisense oligonucleotides loading. Eur J Pharm Biopharm. 2009;71:264-270.

8. Liu X, Howard KA, Donga M, et al. The influence of polymeric properties on chitosan/siRNA nanoparticle formulation and gene silencing. Biomaterials. 2007;28:1280-1288.

9. Khalid MN, Agnely F, Yagoubi N, Grossiord JL, Couarraze G. Water state characterization, swelling behavior, thermal and mechanical properties of chitosan based networks. Eur J Pharm Sci. 2002;15: $425-432$.

10. Farahani TD, Farahani EV, Mirzadeh H. Swelling behaviour of alginate-n, o carboxymethyl chitosan gel beads coated by chitosan. Iranian Polymer J. 2006;15:405-415.

11. Boonsongrit Y, Mitrevej A, Muller BW. Chitosan drug binding by ionic interaction. Eur J Pharm Biopharm. 2006;62:267-274.

12. Hamidi M, Azadi A, Rafiei P. Hydrogel nanoparticles in drug delivery. Adv Drug Deliv Rev. 2008;60:1638-1649.

13. Borges O, Cordeiro-da-Silva A, Romeijn SG, et al. Uptake studies in rat Peyer's patches, cytotoxicity and release studies of alginate coated chitosan nanoparticles for mucosal vaccination. $J$ Control Release. 2006;114;348-358.

14. Ferreira MG, Tillman L, Hardee G, Bodmeier R. Characterization of complexes of an antisense oligonucleotide with protamine and polyL-lysine salts. J Control Release. 2001;73:381-390.

15. Bildestein L, Hillaireau H, Desmaele D, Leptre-Mouelhi S, Dubernet C, Couvreur P. Freeze-drying of squalenoylated nucleoside analogue nanoparticles. Int J Pharm. 2009;381:140-145.

16. Pozo Rodriguez A, Solinis MA, Gascon AR, Pedraz JL. Short and long term stability of lyophilized solid lipid nanoparticles for gene therapy. Eur J Pharm Biopharm. 2009;71:181-189.

17. Guliyeva U, Oner F, Ozsoy S, Haziroglu R. Chitosan microparticles containing plasmid DNA as potential oral gene delivery system. Eur J Pharm Biopharm. 2006;62:17-25.
International Journal of Nanomedicine

\section{Publish your work in this journal}

The International Journal of Nanomedicine is an international, peerreviewed journal focusing on the application of nanotechnology in diagnostics, therapeutics, and drug delivery systems throughou the biomedical field. This journal is indexed on PubMed Central, MedLine, CAS, SciSearch ${ }^{\circledR}$, Current Contents ${ }^{\circledR} /$ Clinical Medicine,

\section{Dovepress}

Journal Citation Reports/Science Edition, EMBase, Scopus and the Elsevier Bibliographic databases. The manuscript management system is completely online and includes a very quick and fair peer-review system, which is all easy to use. Visit http://www.dovepress.com/ testimonials.php to read real quotes from published authors. 